\title{
Visualizing the Evolution of the AI Ecosystem
}

\author{
Rahul C. Basole, PhD \\ Accenture AI \\ rahul.basole@accenture.com
}

\begin{abstract}
This study examines the evolution of the complex, emerging artificial intelligence (AI) ecosystem. Grounded in multiple theories, we introduce a conceptual framework that maps emerging ecosystem dynamics in terms of firm funding and exits. Using a curated dataset of nearly 10,000 ventures and 31,000+ funding/exit activities, we visualize the trajectory of 15 core technology segments of the AI ecosystem as connected scatterplots and compute several salient path measures for each, including path length, velocity, number of loops, and L-shapes. Our visual analysis reveals several path patterns across the four quadrants of our framework and highlights the evolutionary growth and consolidation across segments. We discuss our findings in terms of initial conditions (market size, funding, technology hype), platformication, and geographic concentration. Our study contributes to our data-driven understanding of ecosystem dynamics.
\end{abstract}

\section{Introduction}

The evolution of industries has been a topic of significant interest for many years to both scholars and practitioners [1]. The central premise of these studies has been to explain how, why, when, and where industries have changed or predict what paths they may follow in the future [2]. The resulting insights often influence business strategies, inform investment decisions, or guide policies.

It can be reasonably argued that the study of industry evolution is of particular relevance today. Value creation activities are increasingly interconnected, complex, and global, leading to entirely new forms of organizing [3]. Moreover, rapid technological advances in data, analytics, and artificial intelligence are enabling new business models, fundamentally transforming competitive dynamics, and blurring traditional industry boundaries [4, 5, 6]. Yet, despite their economic importance, understanding of software-centric industry evolution is still limited [7]. Existing theories, models, methods, and findings need to be revisited.

In this study, we focus on the evolution of the emerging artificial intelligence (AI) ecosystem. AI is enabling firms to work smarter and faster, doing more with significantly less, across all enterprise functions $[5,8,9]$. AI is also penetrating many aspects of human lives, from education and health to entertainment and finance [10]. What differentiates AI from other technologies of the past is that AI is seen as a strategic technology by many governments that will have an enormous impact on its economy, society, and security. Countries are thus making significant bets to not only participate but also obtain a major stake in the global AI share $[11,12]$. It is important to note that there is no single industry that defines the AI ecosystem, but rather it is composed of multiple, interconnected enabling technology sub-industries (henceforth referred to as segments) - such as machine learning, robotics, computer vision, or natural language processing. Each of these technology segments will have their own trajectories, but they must be considered in relation to the other segments in order to get a true systemic understanding of AI ecosystem evolution.

This paper makes several contributions. First, we develop a conceptual model of a landscape through which emerging ecosystems can traverse. Second, we visualize the evolutionary paths of segments as connected scatterplots and compute several salient measures. Third, we discuss the findings in terms of initial conditions (market size, funding, technology hype), platformication, and geographic concentration. Theoretically, we advance our understanding of business and entrepreneurial ecosystem dynamics; methodologically, our study contributes to data-driven, visual analytic research of complex ecosystems.

\section{Related Work}

There are several theoretical lenses pertinent to the study of ecosystem evolution. Given its multifaceted 
nature, these lenses can be found in diverse disciplines, ranging from strategy and marketing to technology management and entrepreneurship. A comprehensive review of this literature is beyond the scope of this paper; instead, we opt to highlight key research streams and seminal studies that guided our thinking.

The first research stream includes the consideration of ecosystems as complex systems. In this view, actors across different market segments co-exist, create value, adapt to changes, appear/disappear, and evolve over time [13, 14, 3, 15, 16]. Research in this area primarily focuses on understanding the structure and dynamics of complex systems and is closely related to the field of organization ecology. While the empirical study of ecosystem structure has grown substantially over the past decade [17], research on the dynamics and evolution of ecosystems is still comparatively nascent (see $[18,19]$ for notable exceptions).

The second relevant research stream includes the product life cycle theory, which examines how the pattern of demand growth affects the nature of competition and market maturity over time [20, 21]. The demand-side version of this theory, often depicted as an S-curve, suggests that markets pass through four distinct stages (introduction, growth, maturity, decline). The supply-side version posits that markets evolve until either a dominant product design or scale-appropriability has emerged.

The third research stream is the industry life cycle theory, which describes the evolution of industries from birth to maturity $[21,20]$. It is often divided into stages, such as development, growth, shakeout, maturity, and decline. The transition rate from one stage to another is not uniform and can depend on a number of supply (e.g., technology maturity, competition), demand (e.g., customer preferences), and contextual factors (economic conditions, social, geography, policy, etc.). Industry life cycle theory is concerned with changes in key industry variables, including entry and exit rates, firm numbers, performance and concentration, and in innovative activity as the industry ages [7].

A fourth research stream is the examination of technological hype cycles, which, related to the diffusion of innovations [22], explains a general path a technology takes over time, in terms of expectations or visibility of the value of the technology. Similar to the other foundations, the hype cycle model proposes that technologies progress through successive stages that are pronounced by a peak, followed by disappointment, and later a recovery of expectations [23, 24].

An important stream of research to the study of ecosystem evolution is the consideration of environmental velocity. Environmental velocity considers the rate (e.g., speed, pace, clockrate, etc.) and direction (degree of continuity-discontinuity) of change by one or more dimensions of the organizational environment (demand, competitors, technology, regulation, and products) [25].

The last relevant research stream to the study of ecosystem evolution involves emerging industries. The empirical study of emerging industries has been limited, in particular since it is hard to identify these industries until they mature, many emerging industries can fail, and comprehensive data is limited [26]. Yet, the study of new industries is important, as they have substantial influence on economic growth and employment as well as societal benefits, provide insights into new forms of organizing $[27,26]$.

\section{Conceptual Model}

Following our theoretical foundations, we posit that there are two key activities that characterize the trajectory of emerging ecosystem segments: funding and exits. There are many reasons why entrepreneurial ventures seek funding. For one, funding helps ventures get off the ground and scale their business. It also increases visibility and attracts attention of the market. Broadly considered, the goals of a startup are to survive, scale, and eventually grow to state where it can become a market leader, become a desirable actor in a merger or acquisition, or go public via an IPO. By juxtapositioning these two dimensions, number of funding activities versus the number of exits, and considering "high" and "low" values for each, a conceptual $2 \times 2$ model for understanding ecosystem evolution can be created (see Figure 1) [28].

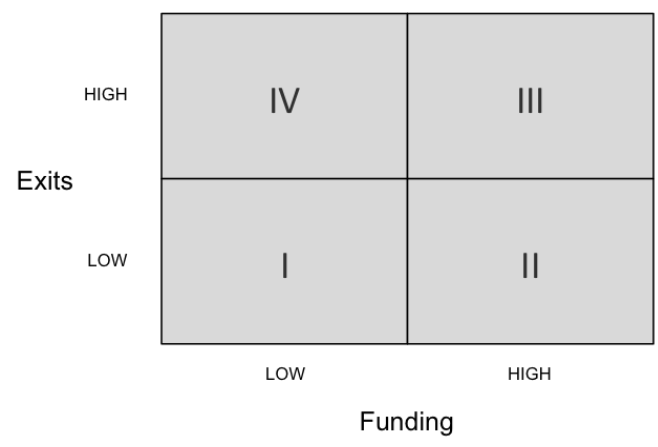

Figure 1: Funding-Exit (F-E) Framework.

\subsection{Quadrant Spaces}

Quadrant I (low funding/low exit) represents the development stage of an ecosystem. The first set of ventures have just formed and are seeking their first 
rounds of funding. The number of actors is still small and there are few competitors. In this stage, the technology hype is likely low and the venture capital community is still seeking to understand and explore the value of the offerings, which are not proven yet, thus being more reserved with their investments. Consequently, since the value of the offerings is not tested, targets are not attractive, thus leading to few mergers and acquisitions. Given the low maturity of the ecosystem, IPOs are also unlikely.

Quadrant II (high funding/low exit) represents the growth stage of an ecosystem where entrepreneurial ventures continue to emerge and funding levels increase significantly. The value of the offerings is better understood and more broadly adopted, thus also garnering interest by investors. The positive levels of funding attracts additional ventures, leading to increased competitive dynamics.

Quadrant III (high funding/high exit) is the most dynamic stage of an ecosystem. It is characterized by significant technology hype, leading to continued increased interest by the investment community. Competitive dynamics are the highest and it is a target rich environment for M\&As. Given the heightened maturity of the ecosystem, some ventures are more established and have the greatest potential to go IPO. In this stage, the ecosystem is healthy and growing.

Quadrant IV (low funding/high exit) represents the consolidation stage of an ecosystem. Technology hype is not high or on the decline as the investment community reduces their funding activities. Yet, established ventures are still attractive targets. Competitive dynamics are still high but consolidation occurs, with incumbents seeking to expand their portfolio.

\subsection{Hypothesized Trajectories}

We can envision several hypothetical trajectories through the F-E framework. One common transition path will begin in (I) and then gradually shift to (II) as the hype builds and segment matures. Segments are unlikely to stay in (II) for a significant time, as one can argue that it is not sustainable to have substantial funding activity without an increase in exits. Segments

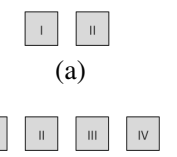

(d)

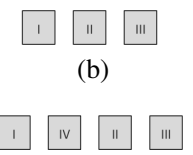

(e) (c)

Figure 2: Hypothesized Trajectories

that advance to the peak and slope stage of the hype cycle will shift to (III), those at the through stage will transition back to (I). As technologies lose their hype and momentum, and fewer new ventures emerge, industries likely shift to (IV). Segments experiencing hypergrowth may transition directly from (I) to (III). Target rich segments may transition from (I) to (IV). When a technology does not evolve as rapidly or generate sufficient hype, it is also likely that paths remain idle in (I). Figure 2 visually summarizes some of the most plausible paths.

\section{Methodology}

\subsection{Data}

We used Crunchbase $^{1}$ as the primary data source for our study. Crunchbase is a widely used socially-curated and expert-validated data source containing comprehensive information about entrepreneurial ventures [29]. Since our focus was on lifecycle of the AI ecosystem, we needed to identify relevant industries. Crunchbase organizes their data, among many other factors, around major industry groups and industries. While there is an artificial intelligence industry category, sole focus on it may potentially be excluding firms in important related spaces. To ensure comprehensiveness, we identified 15 core AI ecosystem segments: analytics (AN), artificial intelligence (AI), big data (BD), business intelligence (BI), database (DB), data integration (DI), data mining (DM), data visualization (DV), image recognition (IR), intelligent systems (IS), machine learning (ML), natural language processing (NLP), predictive analytics (PA), speech recognition (SR), text analytics (TA). Any venture belonging to at least one of these segment categories was included in our initial data extraction. ${ }^{2}$

We constrained our sample by several additional criteria. First, we focused on companies founded between 2010 and 2019. This timeframe allows us to control for firms that are newly formed but had some time to develop, receive funding, and potentially exit. Second, we focused on ventures that had received at least one round of funding (angel, seed, venture series, etc.) and excluded any funding rounds post IPO, M\&A, and close. Third, we focused on for-profit ventures (thus excluding non-profit and social ventures). For-profit ventures are driven by both mission and marketplace. In total, this led to 9,963 ventures, 30,898 rounds of

\footnotetext{
${ }^{1}$ https: / / crunchbase.com

${ }^{2} \mathrm{~A}$ limitation of our data source is that companies may change their segment affiliation over time. Our study currently does not account for this possibility. To overcome this issue, data would have to be extracted over time.
} 
funding, and 779 M\&As and 31 IPOs. We extracted the data using the Crunchbase export functionality and stored it in relational tables for subsequent analysis and visualization.

\subsection{Visualization}

We utilize a connected scatterplot approach to visualize the life cycle paths of AI industries. A connected scatterplot visualizes two related time series in a scatterplot and connects the points with a line in temporal sequences [30]. The technique has been used for many centuries as an analysis tool for time series data, but has seen a particular growth as a communication tool in recent years [30].

The main power of the connected scatterplot is that salient trends are easier to detect, such as positive and negative correlations, which particular time series is changing more quickly, and rapid temporal shifts. The core components of a scatterplot include points (typically shown as dots and circles), where the $x$ and $y$ position denotes the time series values at a given time, lines that connect consecutive points, arrows that indicate the direction time, and selected labels to help orient the readers.

Figure 3 shows a sample connected scatterplot in the context of our study (funding vs. exits) and a rubric in how to read the direction of each segment. In total there are eight different directions that each line segment can take between any two time points. When the line goes to the right, funding is increasing, when it goes left, funding is decreasing; when the line goes up, exits are increasing; when they go down, exits are decreasing. Diagonal lines thus indicate a combination of funding [increase/decrease] and exit [increase/decrease].

When considering the entire linegraph, connected scatterplots can contain two interesting visual features, namely L-shapes and loops. The L-shape feature is characterized by a close to $90^{\circ}$ change in line direction and represent sudden change in the relationships between the two time series. Loops on the other hand indicate a temporal shift between the two series, and indicate the simultaneous appearance of a local maximum and minimum. The direction of the loop can indicate in which time series the pattern first occurs, thus identifying a potential causal relationship. A clockwise loop indicates that time series on the vertical axis starts the patterns, while a counter-clockwise loops indicates that the horizontal axis started it. For more details about connected scatterplots, readers are referred to the study by [30].

Since a connected scatterplot is not an off-the-shelf visualization technique, we implemented a custom visualization using the ggplot 2 tidyverse $^{3}$ library in $\mathrm{R}$. Code is available upon request. Heatmap and histogram visualizations were created in Tableau 2019.1.12 $2^{4}$ The path similarity network was created in $\mathrm{Gephi}^{5}$.

\subsection{Artificial Categorization}

Artificial categorization refers to the process of converting a continuous variable into a categorical one [31]. A key advantage of using artificial categories is that they simplify the interpretations of variables, the analysis, and ultimately presentation of the results. Since our theoretical framework considers a $2 \times 2$ lens of the funding/exit landscape, we must determine appropriate thresholds that split each of our two continuous variables (i.e., funding and exit) into "low" and "high" groups.

One of the most commonly used approaches for dichotomizing a continuous variable is to use a median split. While some have highlighted the weakness of a median split (e.g., Type I error), we believe that it still is well suited for our analysis, as alternate approaches such as a midpoint split, mean split, natural split, or upper/lower quartile or tercile (dropping the middle one(s)) would not make sense given the nature of our data and study context [32].

Since we examine both the overall AI ecosystem and individual segments, we computed the median for both funding and exit associated with each focus context and overlaid these as vertical and horizontal lines on our connected scatterplot visualizations to create the $2 \times 2$ matrix.

\section{Results}

\subsection{Descriptive Analysis}

Figure 4 shows the evolution of venture emergence by AI ecosystem segment. The most startups appear in the AI, analytics, big data, and ML segments; the least number of ventures in our timeframe are in speech recognition and text analytics. The heatmap reveals that all segments had a relatively steady growth from 2010 until 2015/16 and then a gradual and in some instances rapid decline. The figure also shows that segments have different peak years, with AI and ML peaking in 2016, Analytics in 2013, and Big Data in 2014.

One important point that needs to be noted about Figure 4 is the distribution of ventures across segments. Ventures do not always map into a single ecosystem segment. In fact, it is quite likely that ventures may tag

\footnotetext{
${ }^{3}$ https: //ggplot2.tidyverse.org/

${ }^{4}$ https: //www.tableau.com/

${ }^{5}$ https://gephi.org/
} 

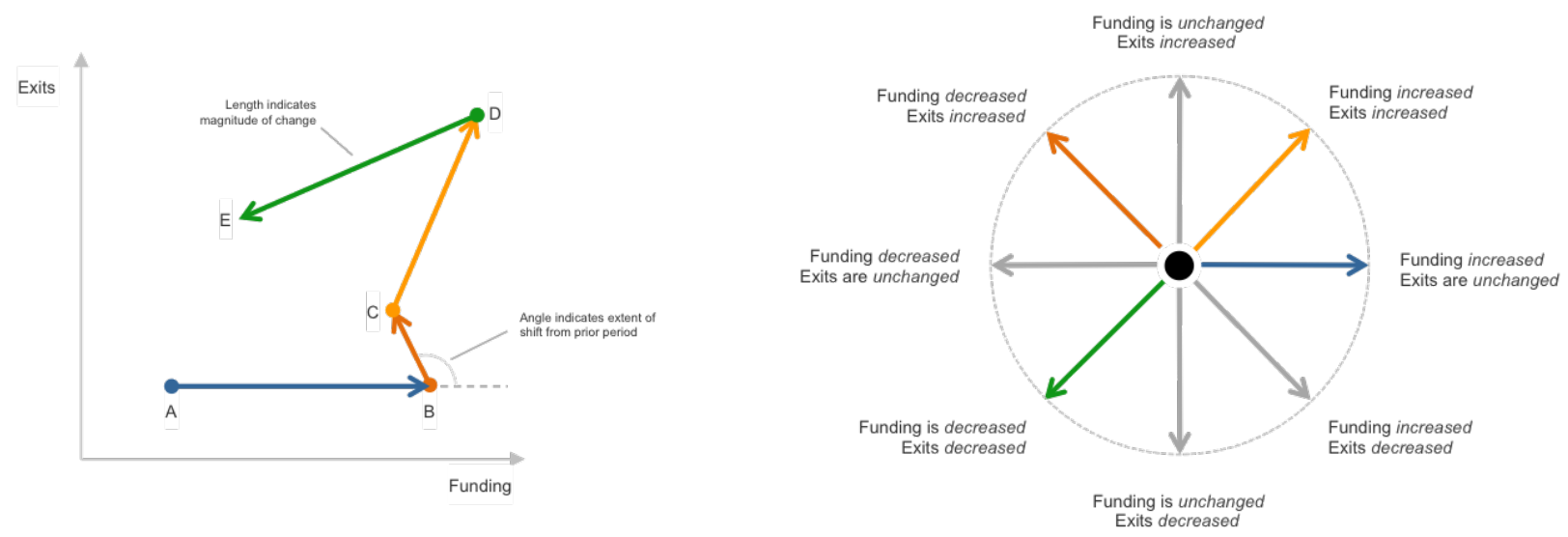

Figure 3: How to read the F-E connected scatterplot.

themselves as operating across two or more segments. Consequently, the sum of totals for each segment is greater than the number of ventures in our sample.

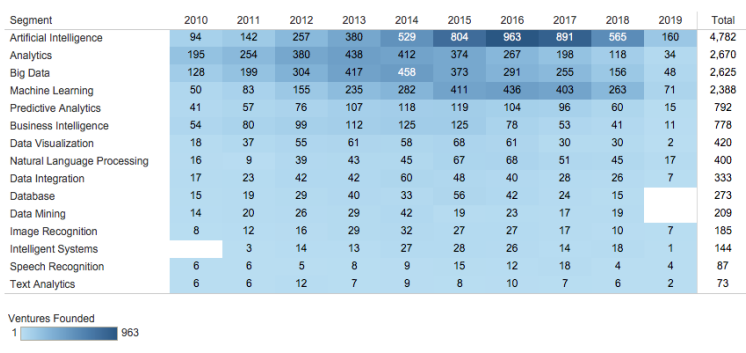

Figure 4: Ventures founded by AI ecosystem segment.

A histogram analysis of segment co-occurrence shows that the majority of ventures belong to 1-2 segments; a much smaller percentage of ventures have more than 6+ categories (see Figure 5).

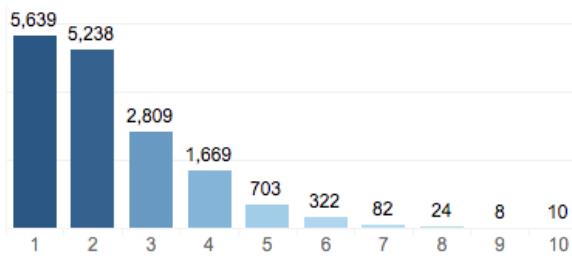

Figure 5: Ecosystem segments distribution.

When analyzing the co-occurrence of two segments for a venture we can observe that $\mathrm{AI}$ and $\mathrm{ML}$ are the most frequent combination with 5,108 ventures (see Figure 6). This is followed by analytics and BD with $(3,176)$ ventures and AI and analytics and ML and analytics, suggesting the relative core aspect of these segments to the ecosystem. The heatmap however also reveals some segments that co-occur infrequently or not at all, such as combination of speech recognition and image recognition ventures, identifying potential opportunities for startup white spaces.

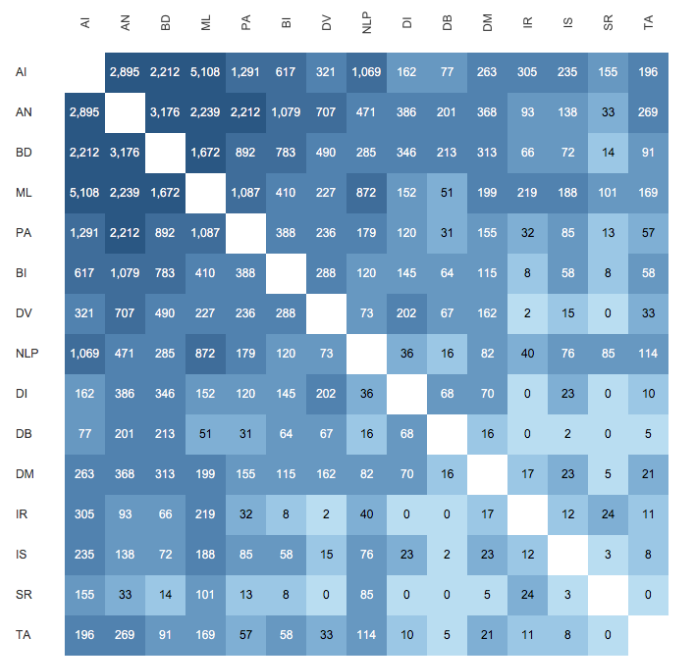

Figure 6: Co-occurrence of AI ecosystem segments.

\subsection{AI Ecosystem Segment Trajectories}

Figure 7 (a)-(o) shows the trajectory of each segment. It should be noted that the scale of each visualization is segment-specific. The visualizations reveal several different path patterns, with some segments covering all quadrants and others only a few. Table 1 provides a complementing summary of each segment's path trajectory including quadrant path, total 


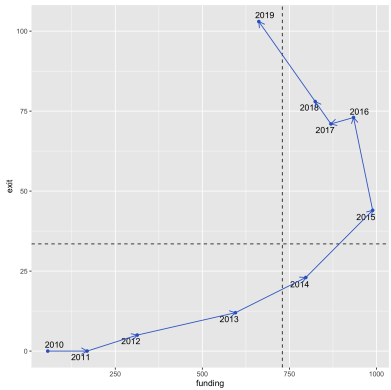

(a) Analytics

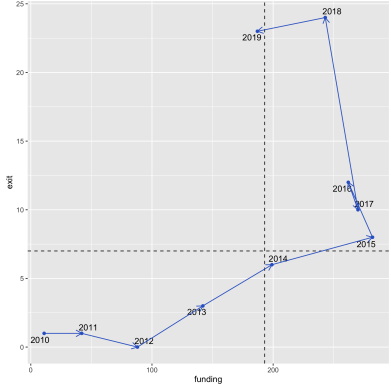

(d) Business Intelligence

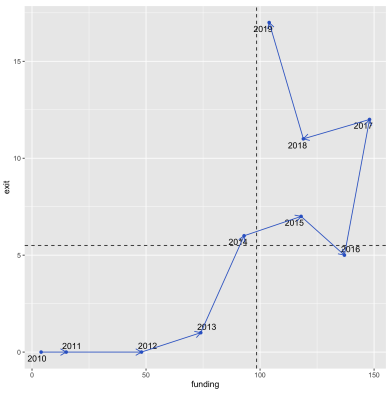

(g) Data Visualization

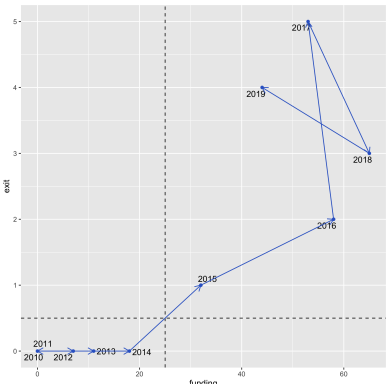

(j) Intelligent Systems

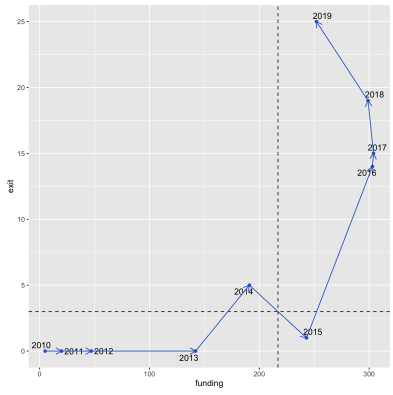

(m) Predictive Analytics

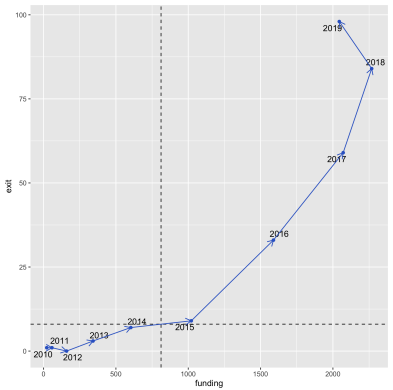

(b) Artifical Intelligence

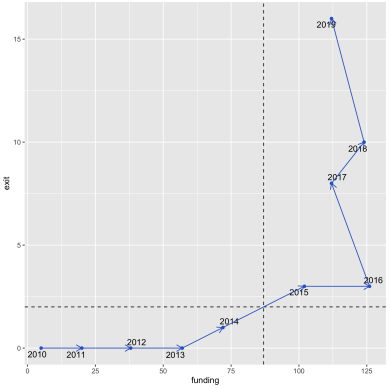

(e) Data Integration

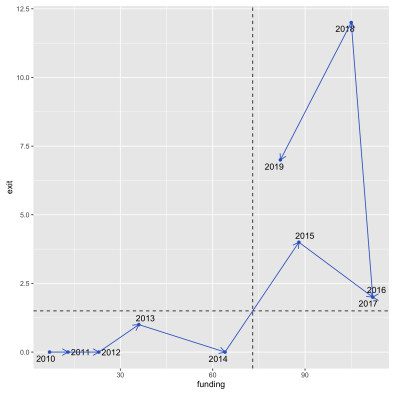

(h) Database

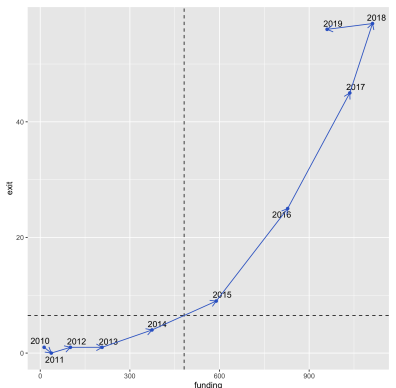

(k) Machine Learning

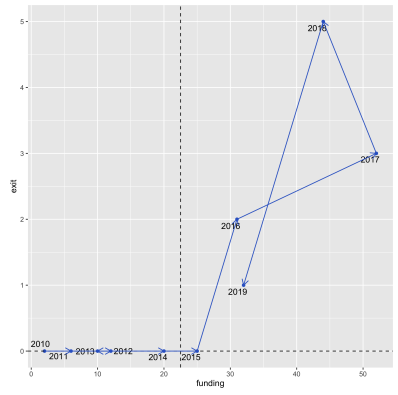

(n) Speech Recognition

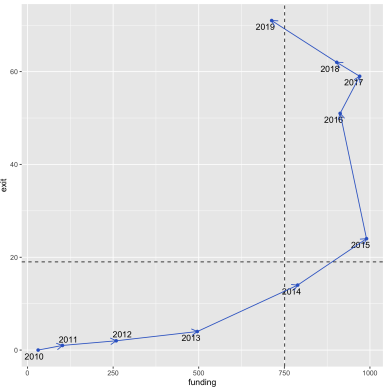

(c) Big Data

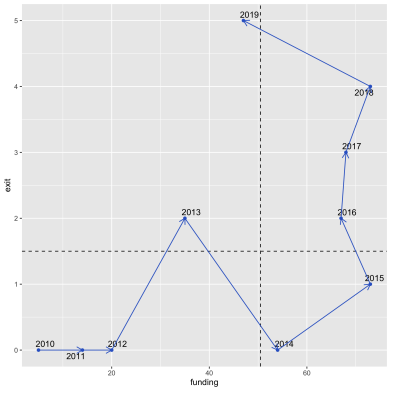

(f) Data Mining

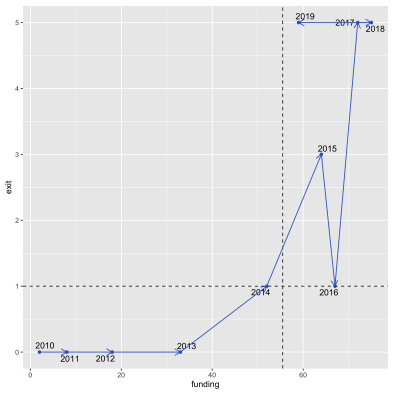

(i) Image Recognition

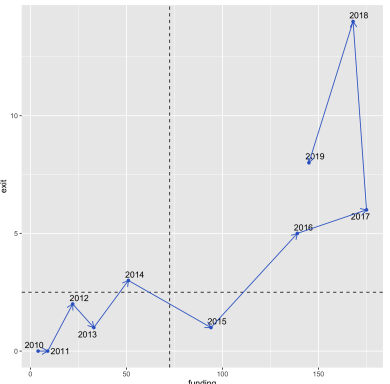

(1) Natural Language Processing

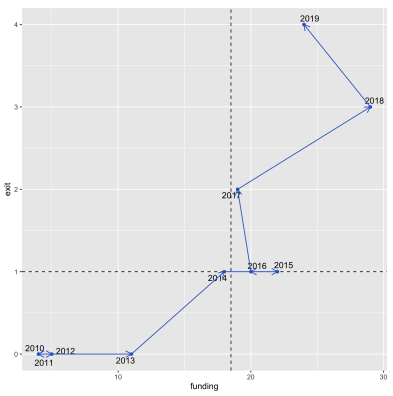

(o) Text Analytics

Figure 7: Trajectories of AI Ecosystem Segments 


\begin{tabular}{|c|c|c|c|c|c|c|c|c|c|c|}
\hline \multirow[b]{2}{*}{ Segment } & \multirow[b]{2}{*}{ Path } & \multirow[b]{2}{*}{ Path Length } & \multicolumn{5}{|c|}{ Velocity } & \multirow[b]{2}{*}{ L-Shapes } & \multirow[b]{2}{*}{ Loops } & \multirow[b]{2}{*}{ Quadrants } \\
\hline & & & Avg. & Std. Dev. & $\operatorname{Min}(\bullet)$ & Max. (•) & Timeline & & & \\
\hline SR & I - II - III & 75.24 & 8.36 & 5.72 & 2.00 & 21.02 & $-\bullet-\bullet$ & 5 & 2 & 3 \\
\hline $\mathrm{BD}$ & I - II - III - IV & 1355.87 & 150.65 & 85.66 & 57.56 & 293.17 & $-\bullet-\bullet-$ & 3 & 0 & 4 \\
\hline AN & I - II - III - IV & 1271.28 & 141.25 & 78.10 & 45.54 & 282.09 & $-\bullet-\bullet-$ & 3 & 0 & 4 \\
\hline BI & I - II - III - IV & 386.26 & 42.92 & 22.70 & 8.25 & 83.02 & $-\bullet-\bullet-$ & 4 & 1 & 4 \\
\hline AI & I - III & 2473.29 & 274.81 & 178.25 & 36.00 & 568.51 & $\bullet-\bullet-$ & 2 & 0 & 2 \\
\hline ML & I - III & 1253.55 & 139.28 & 75.44 & 24.02 & 239.53 & $\bullet--\bullet-$ & 1 & 0 & 2 \\
\hline DI & I - III & 161.55 & 17.95 & 5.76 & 12.17 & 30.07 & $-\bullet-\bullet-$ & 3 & 0 & 2 \\
\hline DB & I - III & 141.21 & 15.69 & 9.68 & 0.00 & 28.02 & $-\bullet-\bullet$ & 5 & 0 & 2 \\
\hline IS & I - III & 97.08 & 10.79 & 8.40 & 0.00 & 26.02 & $\bullet-\bullet-$ & 3 & 1 & 2 \\
\hline IR & I - III & 91.20 & 10.13 & 5.76 & 3.00 & 19.03 & $-\bullet-\bullet-$ & 4 & 1 & 2 \\
\hline TA & I - III & 36.63 & 4.07 & 3.30 & 0.00 & 10.05 & $\bullet-\bullet-$ & 3 & 2 & 2 \\
\hline PA & I - IV - II - III & 355.00 & 39.44 & 29.96 & 1.41 & 95.00 & $-\bullet-$ & 2 & 0 & 4 \\
\hline NLP & I - IV - II - III & 205.95 & 22.88 & 15.02 & 5.00 & 45.18 & $\bullet--\bullet-$ & 6 & 0 & 4 \\
\hline $\mathrm{DM}$ & I - IV - II - III - IV & 106.88 & 11.88 & 8.26 & 1.41 & 26.02 & $\longrightarrow-\bullet$ & 5 & 0 & 5 \\
\hline DV & I - IV - III - II - III & 192.00 & 21.33 & 7.42 & 11.00 & 33.00 & $\bullet-$ & 4 & 0 & 5 \\
\hline
\end{tabular}

Table 1: Trajectory Summary (grouped by path).

path length, average, minimum, and maximum velocity, a temporal depiction when the minimum and maximum velocity occurred, and the number of L-shapes and loops.

All trajectories begin in Quadrant I; most paths remain in (I) for 3-4 years, with the exception of data mining which rapidly jumped into Quadrant IV after only two years. Four out of fifteen segments (SR, BD, AN, and BI) transition from Quadrant I to II. 7 out of 15 segments (AI, ML, DI, DB, IS, IR, and TA) all transition from Quadrant I to III, suggesting that most AI segments have moved to hyper-growth relatively quickly. Four segments (PA, NLP, DM, DV) transitioned to Quadrant IV first, suggesting that ventures in these segments were very attractive targets quickly.

Path length indicates the total distance the segment traversed in the funding-exit landscape. Average velocity indicates how quickly each segment moves from one year to the next. The top four segments for each measure are AI, BD, ML, and AN; the bottom four are TA, SR, IR, and IS. The AI industry is not only moving the fastest on average, but also had the fastest velocity. When considering when both the maximum and minimum velocity occurred, we can see that the maximum velocity succeeded the minimum velocity in 8 out of 15 segments. In only four segments (AN, BI, DI, DB) we observe that the minimum velocity is occurring at the end of our study timeframe. The highest early velocity can be identified in the DV segment.

Considering the two salient visual features of connected scatterplots, we can see that NLP has the most L-shapes with 6 , followed by SR, DB, and DM with 5 . The fewest L-shapes can be observed in the ML segment (1) and AI segment (2). In terms of loops, most segments have no loops. The five exceptions include SR and TA (with 2 loops each) and BI, IS, and IR (with 1 loop each).

\subsection{Path Similarity}

While the paths and path metrics provide important insights into the trajectory of segments, it is unclear how similar these paths are. To understand the similarity between segments paths, we built a comprehensive feature vector of path characteristics. In addition to the metrics shown in Table 1, features included the number of quadrant transitions, types of quadrant transitions, years within each quadrant, and years between minimum and maximum velocity. We then computed the pairwise Euclidean distance between each of the segment path feature vectors and used the inverse to compute segment similarity. Since all segments paths share some similarity, we used a threshold to only identify the most similar segments. Visualizing the path similarity network using a force-directed graph algorithm (OpenORD) and color-encode it by Louvain modularity reveals three distinct path clusters (see Figure 8). Cluster 1 consists of relatively well-established segments (BD, BI, PA, DB, AN, ID, and IR); Cluster 2 consists of DM, DV, NLP, and SR; Cluster 3 consists of AI, ML, IS, and TA.

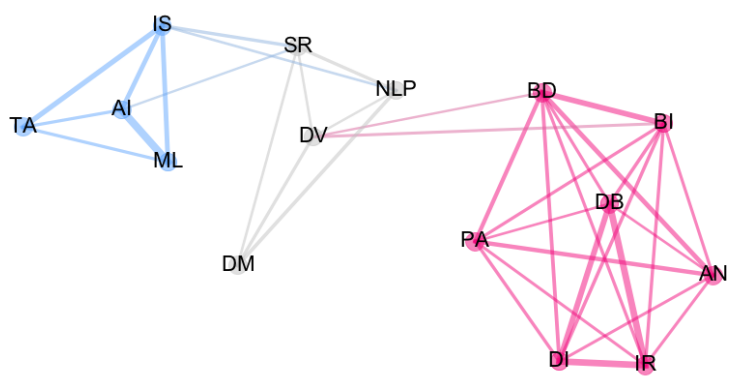

Figure 8: Path Similarity Network. 


\section{Discussion}

Our study makes two important observations. First, when examining ecosystem dynamics it is important to not consider ecosystems as single, homogeneous units, but rather unpack the ecosystem into appropriate segments. Different segments of an ecosystem can evolve differently; results summarized at the global level could thus fail to reveal local variations that likely exist. Second, our analysis shows that while there are differences between segments, some common patterns exist. A quadrant-based view can provide an important macro-perspective, while an examination of path characteristics can provide a more granular view of segment trajectories through the F-E landscape.

The insights from our visual analysis, however, generate an important follow-on question. What factor(s) explain the shape of path trajectories? If we take a complex system lens to ecosystem evolution [13], simple linear explanations of cause-and-effect are likely not sufficient. In fact, it can be reasonably argued that the shape of the curve is more likely a result of multiple interconnected positive and negative reinforcing causal loops mitigated by a variety of factors. Moreover, since ecosystems are not memoryless, initial conditions will likely also matter in determining the trajectory of a segment. A segment that is already doing well, where startups are attracting funding, flourishing, and successfully exiting, will more likely continue to attract interest by founders and investors. Of course this attractiveness is potentially mitigated by the maturity of the technology, competitive dynamics, and other current economic conditions.

When considering the initial conditions of our segments (see Table 2), we can see that the three largest segments pre-2010 were analytics, business intelligence, and big data, with a total of more than 5,000 ventures with nearly $\$ 10 \mathrm{~B}$ in funding. These segments also experienced the greatest number of exits, with 131 acquisitions and 58 IPOs. In terms of technology hype, both analytics and business intelligence were in the latter stages of the hype cycle, while big data was still on the rise. The relative size and well-funded industry may explain the more complete path these segments took across the four quadrants. In contrast, consider the initial conditions of artificial intelligence, intelligent systems, or machine learning. All three of these segments were in the early phases of the hype cycle (on the rise), had a moderate number of ventures $(1,400)$, and still relatively low levels of funding (367 rounds and \$1.8B) and exits. The industry, however, experienced a massive growth in the decade that followed, with trajectories still remaining in Quadrant III.

\begin{tabular}{|c|c|c|c|c|c|c|c|}
\hline & \multirow[b]{2}{*}{ Size } & \multicolumn{2}{|c|}{ — Funding - } & \multicolumn{3}{|c|}{ — Exits — } & \multirow[b]{2}{*}{ Hype Cycle $^{6}$} \\
\hline & & Rnds & Total & Acq & IPO & Cl & \\
\hline $\mathrm{AN}$ & 1,803 & 770 & 5.7 & 66 & 24 & 8 & Trough (2009) \\
\hline $\mathrm{AI}$ & 785 & 190 & 0.8 & 9 & 15 & 2 & Rise (2017) \\
\hline $\mathrm{BD}$ & 1,159 & 354 & 2.7 & 16 & 24 & 5 & Rise (2011) \\
\hline BI & 1,236 & 255 & 2.1 & 44 & 10 & 8 & Plateau (2009) \\
\hline DI & 413 & 110 & 1.1 & 21 & 4 & 1 & Rise (2011) \\
\hline $\mathrm{DM}$ & 191 & 36 & 0.2 & 5 & 4 & 1 & Slope (2009) \\
\hline DV & 337 & 87 & 1.2 & 9 & 3 & 1 & Trough (2009) \\
\hline DB & 725 & 165 & 1.2 & 35 & 5 & 2 & Plateau (2012) \\
\hline IR & 136 & 50 & 0.2 & 7 & 3 & 1 & Peak (2011) \\
\hline IS & 122 & 42 & 0.3 & 7 & 4 & 3 & Rise (2013) \\
\hline ML & 514 & 135 & 0.7 & 6 & 8 & 2 & Rise (2014) \\
\hline NLP & 112 & 45 & 0.3 & 5 & 0 & 0 & Slope (2010) \\
\hline $\mathrm{PA}$ & 256 & 93 & 0.5 & 3 & 4 & 0 & Slope (2010) \\
\hline SR & 71 & 24 & 0.2 & 8 & 1 & 1 & Slope (2010) \\
\hline TA & 67 & 27 & 0.1 & 2 & 1 & 0 & Trough (2009) \\
\hline
\end{tabular}

Table 2: Initial Conditions of AI Segments (pre-2010).

While initial conditions influence the shape of the path, following principles of complex systems (emergence, non-linear, adaptive, self-organizing, and stochastic), industries will continually evolve and adapt to contextual conditions. Prior work has shown that there can be a myriad number of influencing factors, including technological (e.g., maturity/readiness), economic (e.g., competitive dynamics, venture capital access), organizational (e.g., leadership, talent), social (e.g., market demand/preferences), or regulatory (e.g., policy). Consequently, to fully explain the trajectory of different industries will require further empirical exploration of these factors, which is beyond the scope of this paper. Instead, we frame our findings on two increasingly debated topics shaping AI industries today: platformication and geographic concentration.

\subsection{Platformication}

Platforms have been shown to be an important driver of economic growth in a wide range of industries [4]. Platforms play a particularly important role in software-centric industries, where value is created by connecting and enabling a wide range of stakeholders [33]. There are many benefits of being a platform business, including the ability to grow faster and scale better, leverage the power of the community to innovate and create value, and achieve greater efficiencies. Some of the most successful organizations today operate a platform business model.

Given the success of platform companies, it can be reasonably argued that the venture capital community

\footnotetext{
${ }^{6}$ We utilized multiple hype cycles to identify the stage of each segment, including the Hype Cycles for Emerging Technologies (2010, 2011), Business Intelligence and Performance Management (2009), Big Data (2012), Embedded Software and Systems (2013), Advanced Analytics and Data Science (2014), and Artificial Intelligence (2017).
} 

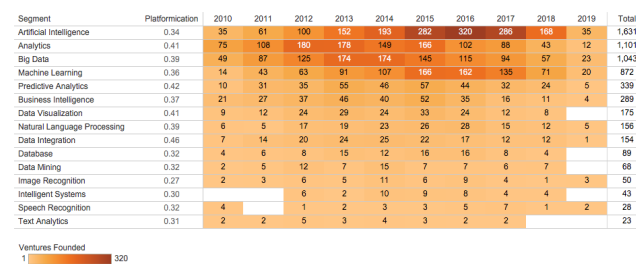

(a) Platformication of Segments

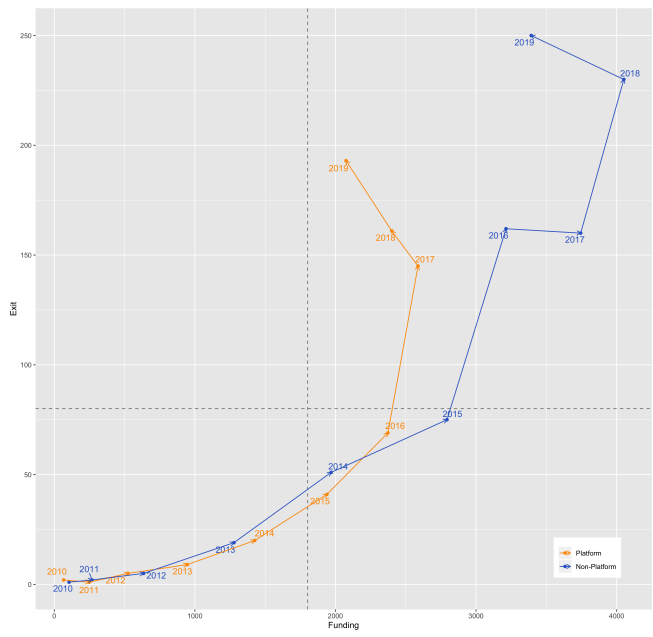

(b) Path Comparison

Figure 9: Analysis of Platform Ventures

as well as potential acquirers would value firms with platform capabilities. Consequently, we would expect that the trajectories of platform ventures would be somewhat different than those of non-platform companies across segments.

Figure 9 shows the segment platformication ${ }^{7}$ over time, and the comparative F-E trajectory. ${ }^{8}$ We can see that all segments have a platformication of greater than $27 \%$, with the highest level in data integration (46\%) and predictive analytics (42\%) and the lowest in image recognition $(27 \%)$. The trajectory also shows some differences between platform and non-platform companies, with platform companies experiencing a significant jump in exits between 2016-17 compared to non-platform companies.

\subsection{Geographic Concentration}

The race for AI dominance is not confined to a single country. While the U.S. and China have the largest and

\footnotetext{
${ }^{7}$ We used text mining applied to the business description of each company to determine if they were a platform venture. We then computed the ratio of platform ventures to all ventures in a segment to compute platformication index.

${ }^{8}$ Due to space constraints, we only show an aggregate AI ecosystem comparison. Segmental comparison shows the divergent paths more clearly.
}

most well-funded companies, many other countries are making significant AI investments as well [12]. Global competition can thus also influence the shape of the trajectory. Using the 26 countries included in the Global AI Vibrancy analysis of the 2019 AI Index Report [12], we compare the geographic concentration of ventures founded in a segment-year and the country-level paths of AI ventures in the top six countries (see Figure 10).

The visual analysis reveals that the average concentration ranges from lowest in image recognition $(0.67)$ to highest in data visualization (0.85). In some years, in fact, there are some years and segments in which there is tremendous geographic concentration (e.g., intelligent systems in 2019). The results clearly show that the top six countries are very much dominating the AI race in terms of founding of new ventures, suggesting potentially favorable resources, ecosystem, and policies in place. The path comparison in Figure 10(b) reveals that in fact the U.S. stands out significantly from the other five countries, but almost all trajectories are following a hypergrowth path.

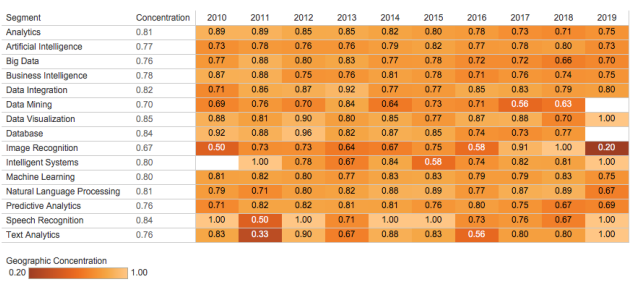

(a) Geographic Concentration of Segments

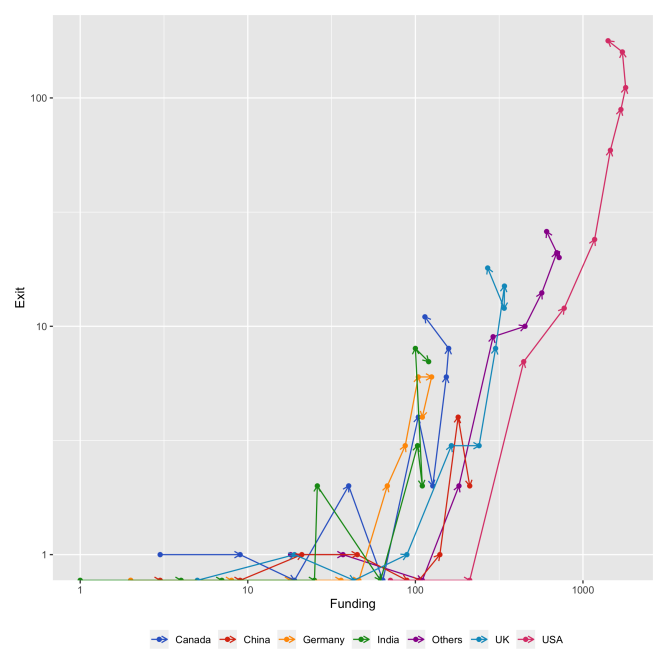

(b) Path Comparison

Figure 10: Geographic Analysis 


\section{Concluding Remarks}

This study provides a visual analysis of the evolutionary dynamics of the AI ecosystem. We frame our thinking in terms of a temporal path through a conceptualized funding-exit (F-E) landscape. Our investigation reveals differences across ecosystem segments but also identifies some common patterns. The study is merely an initial step and we hope it will stimulate future research.

\section{References}

[1] F. Malerba, R. R. Nelson, L. Orsenigo, and S. G. Winter, Innovation and the Evolution of Industries: History-Friendly Models. Cambridge University Press, 2016.

[2] A. M. McGahan, How industries evolve: Principles for achieving and sustaining superior performance. Harvard Business Press, 2004.

[3] M. Iansiti and R. Levien, The keystone advantage: what the new dynamics of business ecosystems mean for strategy, innovation, and sustainability. Harvard Business Press, 2004.

[4] G. G. Parker, M. W. Van Alstyne, and S. P. Choudary, Platform Revolution: How Networked Markets Are Transforming the Economy? and How to Make Them Work for You. WW Norton \& Company, 2016.

[5] M. Iansiti and K. Lakhani, Competing in the Age of Artificial Intelligence. Harvard Business Press, 2020.

[6] A. McAfee and E. Brynjolfsson, Machine, platform, crowd: Harnessing our digital future. WW Norton \& Company, 2017.

[7] M. Peltoniemi, "Reviewing industry life-cycle theory: Avenues for future research," International Journal of Management Reviews, vol. 13, no. 4, pp. 349-375, 2011.

[8] W. B. Rouse and J. C. Spohrer, "Automating versus augmenting intelligence," Journal of Enterprise Transformation, pp. 1-21, 2018.

[9] E. Brynjolfsson and A. Mcafee, "The business of artificial intelligence," Harvard Business Review, pp. 1-20, 2017.

[10] P. R. Daugherty and H. J. Wilson, Human+ machine: reimagining work in the age of AI. Harvard Business Press, 2018.

[11] K.-F. Lee, AI superpowers: China, Silicon Valley, and the new world order. Houghton Mifflin Harcourt, 2018.

[12] R. Perrault, Y. Shoham, E. Brynjolfsson, J. Clark, J. Etchemendy, B. Grosz, T. Lyons, J. Manyika, S. Mishra, and J. C. Niebles, "The AI index 2019 annual report," AI Index Steering Committee, Human-Centered AI Institute, Stanford University, Stanford, CA, 2019.

[13] M. A. Phillips and P. Ritala, "A complex adaptive systems agenda for ecosystem research methodology," Technological Forecasting and Social Change, vol. 148, p. 119739, 2019.

[14] W. B. Arthur, The economy as an evolving complex system II. CRC Press, 2018.

[15] J. F. Moore, "Predators and prey: a new ecology of competition," Harvard Business Review, vol. 71, no. 3, pp. 75-86, 1993.
[16] M. G. Jacobides, C. Cennamo, and A. Gawer, "Towards a theory of ecosystems," Strategic Management Journal, vol. 39, no. 8, pp. 2255-2276, 2018.

[17] K. Järvi, S. Kortelainen, et al., "Taking stock of empirical research on business ecosystems: a literature review," International Journal of Business and Systems Research, vol. 11, no. 3, pp. 215-228, 2017.

[18] R. C. Basole, M. G. Russell, J. Huhtamäki, N. Rubens, K. Still, and H. Park, "Understanding business ecosystem dynamics: A data-driven approach," ACM Transactions on Management Information Systems (TMIS), vol. 6, no. 2, pp. 1-32, 2015.

[19] S. J. Mäkinen and O. Dedehayir, "Business ecosystems' evolution-an ecosystem clockspeed perspective," in Collaboration and competition in business ecosystems, Emerald Group Publishing Limited, 2014.

[20] J. M. Utterback and W. J. Abernathy, "A dynamic model of process and product innovation," Omega, vol. 3, no. 6, pp. 639-656, 1975.

[21] S. Klepper, "Industry life cycles," Industrial and Corporate Change, vol. 6, no. 1, pp. 145-182, 1997.

[22] E. M. Rogers, Diffusion of innovations. Simon and Schuster, 2010

[23] H. Van Lente, C. Spitters, and A. Peine, "Comparing technological hype cycles: Towards a theory," Technological Forecasting and Social Change, vol. 80, no. 8, pp. 1615-1628, 2013.

[24] O. Dedehayir and M. Steinert, "The hype cycle model: A review and future directions," Technological Forecasting and Social Change, vol. 108, pp. 28-41, 2016.

[25] I. P. McCarthy, T. B. Lawrence, B. Wixted, and B. R. Gordon, "A multidimensional conceptualization of environmental velocity," Academy of Management Review, vol. 35, no. 4, pp. 604-626, 2010.

[26] D. P. Forbes and D. A. Kirsch, "The study of emerging industries: Recognizing and responding to some central problems," Journal of Business Venturing, vol. 26, no. 5, pp. 589-602, 2011.

[27] J. C. Picken, "From startup to scalable enterprise: Laying the foundation," Business Horizons, vol. 60, no. 5, pp. 587-595, 2017.

[28] A. Lowy and P. Hood, The power of the $2 \times 2$ matrix: Using $2 \times 2$ thinking to solve business problems and make better decisions. John Wiley \& Sons, 2011.

[29] R. C. Basole, "Understanding ecosystem data," in Proceedings of the 53rd Hawaii International Conference on System Sciences, 2020.

[30] S. Haroz, R. Kosara, and S. L. Franconeri, "The connected scatterplot for presenting paired time series," IEEE Transactions on Visualization and Computer Graphics, vol. 22, no. 9, pp. 2174-2186, 2015.

[31] D. D. Rucker, B. B. McShane, and K. J. Preacher, "A researcher's guide to regression, discretization, and median splits of continuous variables," Journal of Consumer Psychology, vol. 25, no. 4, pp. 666-678, 2015.

[32] R. C. MacCallum, S. Zhang, K. J. Preacher, and D. D. Rucker, "On the practice of dichotomization of quantitative variables.," Psychological methods, vol. 7, no. 1, p. 19, 2002

[33] M. de Reuver, C. Sørensen, and R. C. Basole, "The digital platform: a research agenda," Journal of Information Technology, vol. 33, no. 2, pp. 124-135, 2018. 